\title{
Geo-electrical Prospecting by A.C. Bridge Methods.*
}

By A. B. Bro ughton EdGe.

WLECTRICAL methods of exploring for mineral or A for investigating geological structures are numerous, but with few exceptions they fall into two groups, according to the manner in which the ground and the concealed conductive bodies lying within it are excited, and how the resulting electrical field is investigated.

(i.) Surface Potential Methods.-In these, direct or alternating current is passed conductively through the ground between earthed electrodes spaced up to a mile or more apart. The prospecting operations consist in determining the resulting distribution of potential in the ground and in interpreting the geological significance of any anomalies that are recorded.

(ii.) Electromagnetic Methods.-In these, the ground is excited inductively by means of loops of insulated wire suitably disposed on the ground surface and carrying alternating current, usually at a frequency between 200 and 60,000 cycles per second. The survey is then carried out with portable search coils, by means of which investigations are made of any anomalies that appear in the magnetic field.

The object of the present article is to describe two methods of using a form of A.C. bridge (ratiometer) for electrical prospecting purposes, one of which comes under the first and the other under the second of the two groups that have been defined above. The general principle, which is that of the ordinary A.C. bridge, was first applied by the author in 1925 to surface potential surveys carried out in Rhodesia. The method was afterwards used in Australia by the Imperial Geophysical Experimental Survey, and from it the A.C. potential ratio method and instruments in the forms described below were developed.

\section{For Determining Surface Potentials.}

In order to explain the advantages of the A.C. potential ratio method it is necessary to refer to the well-known equipotential line system which, hitherto, has been the only generally known means of investigating A.C. surface potentials. Although the tracing of equipotential lines over the surface has the advan-

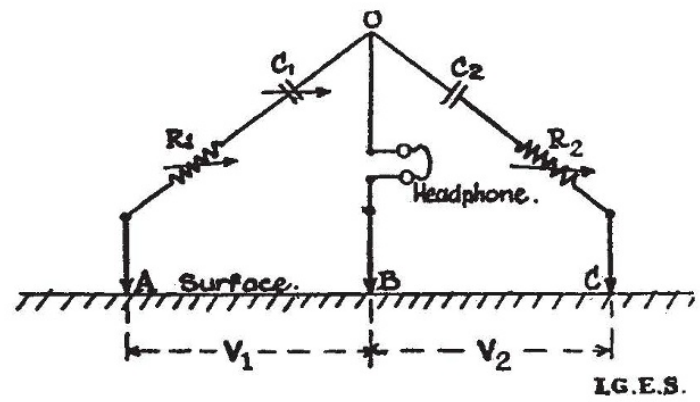

FIG. 1.-Principle of A.C. potential ratio method.

tage of simplicity, it also has a serious defect, since it takes no account of the complex phase conditions that arise when alternating current is applied to ground of variable conductivity. In such circumstances the resultant field has an elliptically polarised structure, in which true equipotential points can only have an instantaneous existence. Although the con-

* Detailed descriptions of these methods and the apparatus employed will be included in the forthcoming publication of the Imperial Geophysical Experimental Survey, entitled "Principles and Practice of Che approval of the Geophysical Executive Committee.

No. 3192, VoL. 127] ception of equipotential lines in such an alternating field is theoretically unsound, the out-of-phase effects met with in practice are not so great as a rule as to preclude the mapping of lines from which a general idea of the conductivity conditions can be obtained. It often happens, however, that a survey of this kind becomes a very slow and inexact performance, and sometimes, when in the neighbourhood of important conductive bodies, the phase conditions are such that the method breaks down completely.

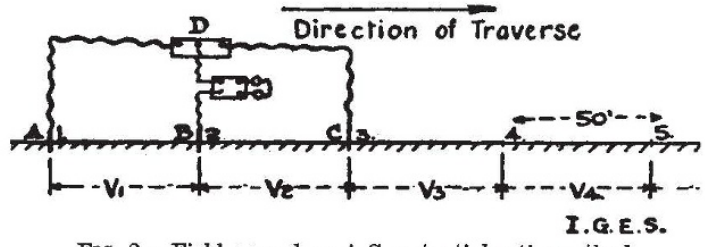

FrG. 2.-Field procedure A.C. potential ratio method.

The fact that this system becomes increasingly inaccurate as a buried conductor is approached is a sufficient indication that an additional surface potential method is required, by which the phase anomalies, which themselves are of diagnostic value, may be properly determined. The A.C. potential ratio method described below is intended to fulfil this purpose.

The principle of the method is shown in Fig. 1, in which $A, B$, and $C$ are contacts made with the ground and $A O$ and $C O$ are ratio arms in each of which a condenser and a resistance are connected. Alternating current, usually at about 500 cycles per second, is passed through the ground between distant earthed electrodes. The purpose of the device is to compare the potential drops $V_{1}$ and $V_{2}$ between the equidistant pairs of contacts $A B$ and $B C$, and to determine the difference in phase angle between them. 'To accomplish this, the capacity and resistance in each arm are adjusted until an exact balance is indicated by silence in the headphones. The potential drops $V_{1}$ and $V_{2}$ will then be directly proportional to the total impedances $\dagger$ in the arms $A O$ and $O C$ respectively, or in terms of the resistances $R_{1}$ and $R_{2}$, and the capacitative reactances $X_{1}$ and $X_{2}$, which are read directly from the instrument.

and

$$
\begin{gathered}
V_{2}=\frac{R_{2} \sin \tan ^{-1}\left(R_{1} / X_{1}\right)}{R_{1} \sin \tan ^{-1}\left(R_{2} / X_{2}\right)} \cdot V_{1} \\
\theta_{2}-\theta_{1}=\tan ^{-1} R_{2} / X_{2}-\tan ^{-1} R_{1} / X_{1},
\end{gathered}
$$

where $\theta_{2}-\theta_{1}$ is the difference in phase angle between $V_{2}$ and $V_{1}$; being of positive value when $V_{2}$ is leading $V_{1}$.

In Fig. 1 the capacity and resistance in each arm are connected in series, but other combinations may be used. As a result of a recent suggestion by D. C. Gall, an instrument is now being made in which a variable resistance alone is included in one arm of the bridge, the other arm containing a fixed resistance and variable condenser connected in parallel. By this arrangement a larger phase angle range is available and the advantage of direct reading is obtained. In the previous designs a somewhat tedious series of computations is necessary in order to obtain the potential ratios and differences in phase angle.

As a rule, the field observations are made along

+ These should be of the order of 100,000 ohms. Otherwise the accuracy of the determination may be seriously affected by the unknown contact resistances at $A$ and $C$ which enter into the measure- 
straight lines, in the manner shown in Fig. 2 ; the station interval depending upon the nature of the problem under investigation but usually being about 50 feet.

The apparatus consists of three steel rods $A, B$, and

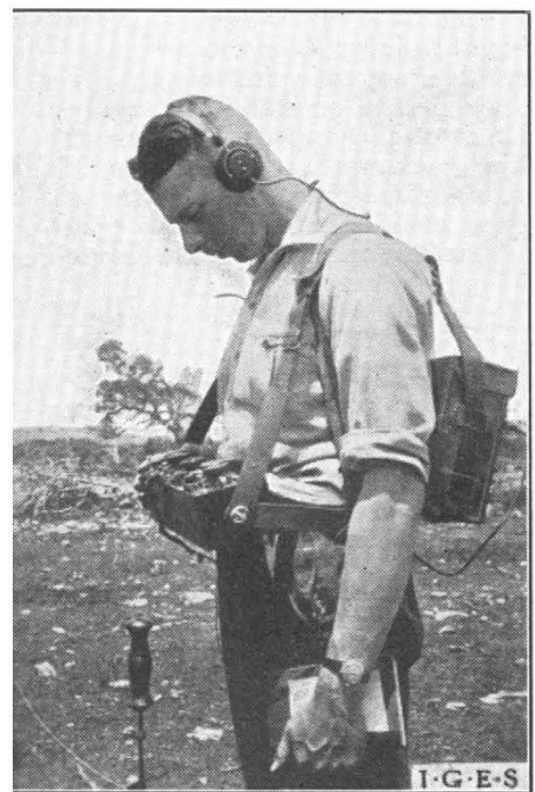

FIG. 3.-Ratiometer in use with amplifier on the operator's back.

$C$, driven into the ground and connected to the ratiometer $D$, which is carried by the operator together with the headphones and amplifier as shown in Fig. 3. Observations are made along the length of the traverse, the operator and his assistants moving forwards one station at a time, so that ratios are obtained for every successive pair of stations. In the case shown in Fig. 2, $V_{1}$ would be taken as an arbitrary unit and $V_{2}, V_{3}, \ldots$. . etc., would then be referred to it by successive multiplication of the individual ratios $V_{2} / V_{1}, V_{3} / V_{2}, \ldots$, etc.* A potential anomaly curve may then be constructed by plotting these multiplied ratios above the mid-points of the station intervals to which they refer. The differences in phase angle are summed consecutively, positive or negative as the will be met with beyond which it is impossible to proceed in the ordinary way. This difficulty may be overcome by side-stepping and including one or more stations off the line of the traverse, but with a bridge of a somewhat different design, now under construetion, these deviations should no longer be necessary.

In many cases it is sufficient to carry out a series of selected straight-line traverses over the ground to be examined and to prepare potential and phase variation curves in the manner indicated above. If a more systematic survey is required, observations must be made along a network of lines so that potential and phase values may be assigned to each station within the area. These values are determined by vectorial addition and, after adjustment of closing errors, equipotential and iso-phase lines may be interpolated.

Field experience in Australia has shown that variations in ground conductivity are recorded very faithfully by this method. In favourable circumstances the potential curves exhibit a well-defined peak over each wall of a buried conductor, and with further research it is thought that the corresponding phase anomalies will be found to have a still greater diagnostic value. In some districts in which marked variations occur in the surface conductivity, the method may be at a disadvantage unless the features to be investigated are of an outstanding character. It is of particular service, however, as an auxiliary to the equipotential line method which, although of admitted value for reconnaissance purposes, is too crude for detailed investigations.

\section{For Comparison of Electromagnetic Fields.}

This application of the ratio arm bridge relates to the simultaneous comparison of magnetic fields at different points in the neighbourhood of conductive bodies, and the case of the vertical components at two points $A$ and $B$ is illustrated in Fig. 4. The two identical coils $C_{1}$ and $C_{2}$, each having an area of several square feet, are supported in a horizontal position immediately above the points $A$ and $B$ and are connected together so that the e.m.f.'s induced in them will assist one another. The ratiometer is then inserted into the circuit in the manner shown and is operated in precisely the same way as in the surface potential application of the instrument. By this means the field intensities may be compared and the differences in phase angle determined. Similar observations may

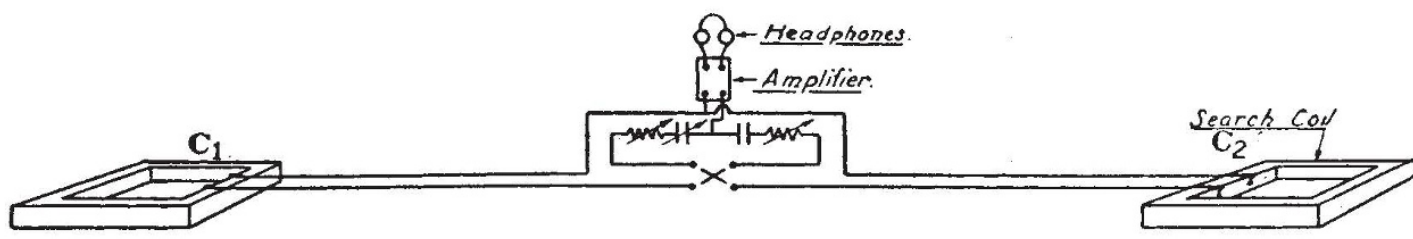

A

B I.G.E.S.

FIG. 4.-Comparison of magnetic fields with the ratiometer

case may be, and are plotted in a similar manner as a phase variation curve.

It will have been observed that in order to obtain a balance with the bridge it is essential that the earth at contact $B$ should be intermediate in potential with respect to the contacts at $A$ and $C$. It may happen, therefore, that even on a straight-line traverse, points

* This multiplication is of complex numbers, that is, $V_{2} / V_{1}$ and $V_{3} / V_{2}$ are multiplied to obtain the magnitude of the product, and their phase angles are added to obtain the phase of the product. be made for other positions of the coils and, in fact, the apparatus may be used in various ways for investigating the character of the ellipse at any point, provided that the differences in phase angle do not lie outside the range of the bridge. It has been pointed out by D. C. Gall that in such cases a mutual inductance bridge would be more satisfactory and such an instrument is now under construction.

As a rule, the field procedure and plotting of intensity and phase variation curves will be carried out 
in the manner already described for the surface potential method, but lines of equal magnetic intensity and iso-phase lines may also be drawn.

The method described above resembles the two-coil balancing system due to Sundberg and Lundberg, in which two similar coils are joined in opposition, with an amplifier and telephone included in the circuit. In this case the relative strengths of the vertical fields are given by the secant of the angle through which one of the coils must be tilted out of the horizontal in order to produce a minimum of sound in the telephone. It has been pointed out by Eve and Keys,* that this procedure is not always satisfactory owing to phase difference in the currents induced in the two coils, and the fact that the tilting of one of the coils will usually introduce a horizontal component into the determination. These writers actually suggest a capacitance bridge in this connexion, but fear that such an instrument would prove too cumbersome for use under ordinary field conditions.

* “Applied Geophysics”, p. 122 (University Press, Cambridge, 1929).

\section{Nomenclature at the Eleventh International Zoological Congress.}

THE account of the International Congress of Zoologists at Padua, published in NATURE for Sept. 27, 1930 (pages 489-490), contained only the briefest reference to the discussions and conclusions on nomenclature. The following account, which is strictly unofficial, may be of interest.

The International Commission on Zoological Nomenclature consists of eighteen members, distributed so far as is practically convenient throughout the world. Most of its work is conducted by correspondence, the intermediary being the secretary. The present secretary is in Washington and is permitted by the U.S. Government to avail himself of official facilities.

The commissioners meet in person at the International Congresses of Zoologists, which hitherto have been triennial, except for an interval from 1913 (Monaco) to 1927 (Budapest), but which by a resolution at Padua are henceforward to be quinquennial. Hitherto each commissioner has been appointed for the triennial period ; whether this will now be changed for a quinquennial period remains to be seen.

Certain changes in the mode of appointment were accepted by the Padua Congress. To replace the retiring commissioners, who are eligible for reappointment, the Commission, after considering the balance of subjects and of geographical regions, submits names to the Section of Nomenclature. The Section can propose other names. The nominees of both Commission and Section are referred to the Permanent Committee of the Congress, which makes the selection and reports it to the plenum of the Congress for confirmation.

The commissioners assemble a week before the Congress opens, and work all day and every day at final discussion and voting on the questions raised during the interval. These may be either opinions on points of nomenclature or proposals for alteration of the rules.

Opinions are settled by a majority vote of the Commission, and this is generally obtained by correspondence.

Proposed changes in the rules must also have received a preliminary majority vote of the whole Commission, that is, ten out of eighteen, and must then receive the unanimous vote of those present at the Congress, nine being a quorum. It follows that, on one hand, eight commissioners may have opposed a proposal in writing, but that nine present in person may carry it; and on the other hand, that seventeen may have been in favour, but that one if present may block it. These are extreme suppositions, but in actual practice the situation is no better, because of the difficulty of bringing all the members together, or even of obtaining a quorum of the actual commissioners. To overcome this difficulty it has been the custom to appoint substitutes (alternates). for the absent commissioners from among zoologists attending the Congress. Thus many of those who actually vote may have an imperfect understanding of the questions at issue. At Padua the preliminary work was done by the chairman (K. Jordan), the secretary (C. W. Stiles), and three other commis sioners ; two more commissioners arrived later, but a quorum was not formed until alternates were appointed. Of these there were no less than eleven, and among them Lt.-Col. J. Stephenson, Mr. G. C. Robson, and Mr. H. W. Parker acted for commissioners in various parts of the British Empire.

On this occasion a further difficulty was due to the fact that before the meeting none of the proposals for change in the rules had received enough votes either to kill it or to bring it up for discussion. Until the Congress assembled it was impossible to get the necessary votes, and then, even if they were obtained, it was too late for adequate and properly informed discussion.

This state of affairs is manifestly undesirable, and for many years considerable dissatisfaction with these arrangements has been expressed, notably with the demand for a unanimous vote by those present. There were two alternative amendments to this bylaw before the Commission, but unfortunately neither received enough preliminary votes to enable it to be discussed.

The same was the fate of other important proposals. Among them were alternative motions which would have had the effect of raising type-designation by elimination from the status of a recommendation to the status of a rule. This is a difficult question, and it is a pity that it could not be thrashed out.

A proposal to revert to the XII. Edition of Linnæus" "Systema Naturæ" as the starting-point of zoological nomenclature was definitely rejected, and the date of the $\mathbf{X}$. Edition was fixed precisely as Jan. 1, 1758, in Article 26 of the rules.

The definition of publication for purposes of systematic zoology was discussed at length, and the conclusions will eventually be submitted in the form of an opinion.

The report of the British National Committee on Entomological Nomenclature was presented by Dr. $K$. Jordan, and its proposed emendations to the rules were considered. A few, of merely editorial nature, were provisionally adopted and others deferred for further consideration.

Proposals that superfamily names based on generic names should all end in -oidea, that new ordinal names should end in ida, and subordinal names in ina were discussed and held for further consideration.

Proposals to apply the law of priority to family and subfamily names, under certain conditions necessitated by other provisions of the Code, were also inevitably held over. The general opinion of the Commission seemed to be against further legislation for groups higher than genera.

As regards homonyms, it was agreed that, ceteris 